\title{
Editorial
}

\section{Air, Hair, Knees, Nose}

\author{
James T. Lee, MD, PhD, FACS
}

\section{One problem with knowing how to hit lots of differ- ent shots is that you will sometimes choose the wrong shot.-Chris Evert}

Surgeons in all specialties use simultaneously an ensemble of multiple infection prevention tactics for every operation under the inspiration that good effects of each tactic will be additive, perhaps even synergistic. This concept of presumed composite efficacy is rarely mentioned in print, or even in polite conversation, but it surely underpins many work steps effected by the nurses and surgeons who currently provide care to nearly 37 million operative patients annually in the United States. A continuing issue is how to decide which tactics we should apply for a given type of operation in the context of surgical-site infection prevention.

In this issue of Infection Control and Hospital Epidemiology, four articles address unresolved issues in surgical-site infection prevention. Sophisticated readers will note that these issues are not "mainstream" in the sense that most of the prevention tactics under discussion are not mentioned often (or at all) in current academic lectures or popular textbooks, nor are they routinely found in guideline-blessed advice. Redirection of attention to less well known prevention tactics, even some "old tricks," is a noticeable characteristic of the oddly amazing resurgence of interest in surgical-site infection prevention and its associated epidemiology projects; one of the problems is identifying which operation types deserve use of "special tactics." Another problem is a potentially serious meta-issue and it has to do with nomenclature; it must be mentioned in summary comments for the current essay.

There is no question that airborne particles contaminated with live bacteria can enter sterile surgical fields during operations. Various methods of reducing that kind of contamination have been toyed with by surgeons for decades. In the modern era, many orthopedic surgeons have become especially fond of using two tactics during joint replacement operations: laminar airflow systems in operating rooms and "space suits" that enclose members of the surgical team in ventilated and exhausted clothing. A third adjunctive tactic, constant ultraviolet irradiation of the operating room environment, has been much less popular. Miner and colleagues provide a nice survey study in which a Medicare database for calendar year 2000 was mined for information about total knee replacement operations. ${ }^{1}$ These authors tallied data for 18,374 operations in 295 hospitals by supplying a 118-item question list to 405 hospitals in four states. Their survey revealed that $30 \%$ of hospitals used laminar airflow for more than $75 \%$ of the operations; $42 \%$ used space suits for the surgical teams; and only $5 \%$ used ultraviolet irradiation in the operating room. Readers should examine this article's rich analytical details carefully. The punch line here is no surprise: there is no clear position, at least in an evidential sense, for the use of laminar airflow or space suits and future workers will need to produce carefully designed and executed clinical trials. Meanwhile, if you need a total knee or total hip operation, it would not be the worst idea to be certain your surgical team uses both laminar airflow and the space suits along with appropriate antimicrobial prophylaxis (proper timing and large dose) and gentle hands on the various tools. One of my pet peeves deserves mention. The authors (who are not surgeons) repeatedly refer to surgeries performed by the or thopedic surgeons. Surgeons perform operations. We do not perform "surgeries" any more than a dermatologist performs "dermatologies" or a hospital pharmacist performs "pharmacies," or a hospital chaplain performs "theologies." This neologism "surgeries" is clearly an affectation that needs to be banned from learned journals. 
Hair is an appendage of skin and its removal from skin at, and around, the expected incision location is one of the oldest, perhaps least understood, and most poorly studied perioperative measures aimed at infection risk reduction. I have been taught that human hair has an intrinsic bacterial population density higher than that of skin in many locations on the body. It is less well known whether modern chemicals we use for conventional antiseptic preparation of the skin before operation also "do their job" adequately on hair surfaces. One thing is certain: it is nearly impossible to establish in a credible, properly designed, clinical trial that hair removal adds benefit. Niël-Weise and colleagues in the Netherlands have provided a thoughtful analysis of randomized clinical trials in which hair removal was studied as an infection risk reduction measure in clean operations. ${ }^{2}$ These authors, participants in the Dutch Working Party on Infection Prevention, surveyed literature up to February 2005, although I could not discern the starting year for that search. After what sounds like an exhaustive and tedious exegesis of the literature, which readers must examine carefully, the authors were able to conclude that "evidence that hair removal has any effect is inconclusive." This may be a disappointment to some in our readership, but it is hardly a surprise to a surgeon. Until there is evidence from a clinical trial, and that is unlikely to occur, most careful and thoughtful surgeons (just the kind you want operating on your husband or wife) will absolutely eschew the razor and use an electric clipper just before skin is prepared. My personal views as a surgeon are that hair removal with clippers has perhaps two, and only two, positive effects: it precludes hair shaft intrusion between skin edges during skin closure and it absolutely assures less discomfort at the 24- to 48hour point when wound dressing tape is removed. Every reader who has not undergone an operation (as I have) will misunderstand this second point.

Most readers of this journal are not surgeons and perhaps would be surprised to learn that all patients bleed in the operating room secondary to operative maneuvers, even if no anticoagulants are "on board." However, it is an unquestioned, Halstedian principle of operating room technique that we must operate in such a way that incisional hematoma frequency postoperatively is as close to zero as possible. There is an abundance of evidence that just a few bacteria in a closed incision pose a greater threat if there is any hematoma present. This phenomenon has been studied for approximately 40 years and, even though the exact mechanism(s) could be described as still uncertain, modern surgical thought is practically unanimous in the teaching that there is benefit to having the subcutaneous space (as well as deeper spaces) "bone dry" at the end of any operation. Now a worrisome complication of orthopedic operations on extremities raises its ugly head in opposition here. It is essentially a standard of care in modern orthopedic work to provide prophylaxis against deep venous thrombosis when a total joint replacement is performed. One of the downside risks is that an incisional hematoma will develop. Asensio and colleagues in Madrid have provided a wonderfully stimulating article that zeroes in on this two-pronged problem from the perspective of infection prevention in knee arthroplasty. ${ }^{3}$ These authors examined the infection frequency for knee replacement operations before and after an institutional policy change that mandated first use of low molecular weight heparin postoperatively instead of preoperatively. Their data are impressive: the infection rate for patients receiving heparin preoperatively was $7.3 \%$. For patients who received their heparin postoperatively, that measure was $1.2 \%$. Readers must carefully read every detail of this study and satisfy themselves regarding the features of the compared patient groups. This is not the first (nor will it be the last) study to confirm an association of incisional infection and incisional hematoma development. ${ }^{4}$ Until new data are in hand, it seems adventuresome to hold the first dose of heparin prophylaxis until the postoperative period for orthopedic patients. In general surgery, we continue to believe that the first dose is optimally used before the operation begins in patients who are "at high risk" for deep venous thrombosis; such patients also have leg compression devices in place and functioning during and after the operation.

An important unresolved question is whether surgical patients should receive mupirocin application preoperatively in the nares as a prophylaxis measure to reduce the likelihood of staphylococcal infection. One subordinate question is which types of operations deserve the boon of this adjunct. Another is whether we should be swabbing the noses of patients preoperatively to identify all Staphylococcus carriers (and thus identify all candidates for mupirocin use). Kallen and coworkers at Dartmouth have generated a partially useful meta-analysis. ${ }^{5}$ This team of investigators examined multiple databases during a 2-month period in 2004, locating 60 pertinent articles. After their application of various selection criteria, only 7 articles survived for what might be called a "mini-meta-analysis." Of this group, 3 were randomized clinical trials and 4 were what the authors call "before-after trials." The study concluded that perioperative use of mupirocin reduces risk of surgical-site infection in non-general surgery cases but not in general surgery cases. Readers must carefully parse every sentence in the Discussion section of this article to glean the salient points. The authors argue that there are few reasons not to use mupirocin, that it is safe, and that patients accept its use. Many readers would recognize the name of a noted academic infectious disease expert from the United States who recently underwent an elective cardiac operation and demanded of his surgeon that this adjunct be used. We definitely need additional clinical trials to confirm that mupirocin use adds benefit, especially in clean category elective operations for patients who are known Staphylococcus carriers.

One of the disappointing side effects of the recent resurgence of interest in surgical-site infections is a meta-problem: very widespread misuse of formal nomenclature. The Centers for Disease Control and Prevention (CDC) defined the concept of "surgical site infection" 13 years ago and it is very clear that the CDC term "surgical site infection" denotes a category of nosocomial infection that contains four types of infection. ${ }^{6}$ Thirteen 
years later, we find misuse of the nomenclature in mainstream journals and this misuse continues at a steady pace. ${ }^{7}$ With the exception of the article by Asensio and colleagues from Madrid, ${ }^{4}$ the other authors mentioned above uniformly fail to inform readers regarding exactly which kind of outcome flaw was under consideration, even though the term "surgical-site infection" (or its abbreviation SSI) is used more than 12 dozen times in total. Potentially four types of infection are implied by the CDC category term "surgical site infection." These are the superficial incisional infections, deep incisional infections, organ infections, and space infections. If we fail as authors to define specifically which of these four types we are referencing in academic work products, future readers of our work will assuredly encounter functionally encrypted messages.

\section{REFERENCES}

1. Miner AL, Losina E, Katz JN, Fossel AH, Platt R. Infection control practices to reduce airborne bacteria during total knee replacement: a hospital survey in four states. Infect Control Hosp Epidemiol 2005;26:910-915.

2. Niël-Weise BS, Wille JC, van den Broek PJ. Hair removal policies in clean surgery: systematic review of randomized, controlled trials. Infect Control Hosp Epidemiol 2005;26:923-928.

3. Asensio A, Ramos A, Múnez E, Vilanova JL, Torrijos P, García FJ. Preoperative low molecular weight heparin as venous thromboembolism prophylaxis in patients at risk for prosthetic infection after knee arthroplasty. Infect Control Hosp Epidemiol 2005;26:903-909.

4. Lee ES, Santilli SM, Olson MM, et al. Wound infection after infrainguinal bypass operations: multivariate analysis of putative risk factors. Surgical Infections 2000;4:257-263.

5. Kallen AJ, Wilson CT, Larson RJ. Perioperative intranasal mupirocin for the prevention of surgical-site infections: systematic review of the literature and meta-analysis. Infect Control Hosp Epidemiol 2005;26:916-922.

6. Horan TC, Gaynes RP, Martone W, et al. CDC definitions of nosocomial surgical site infections, 1992: a modification of CDC definitions of surgical wound infections. Infect Control Hosp Epidemiol 1992;13:606-608.

7. Lee J. Nomenclature nightmare. Surgical Infections 2003;4:293-296. 